\title{
The Influence of Varying the Optical Wavelength on ISL Performance Recognizing High Bit Rates
}

\author{
Asmaa Zaki M. ${ }^{1}$, Heba A. Fayed ${ }^{2}$, Ahmed Abd El Aziz ${ }^{3}$, Moustafa H. Aly ${ }^{4}$ \\ Photonics Research Lab (PRL) \\ ${ }^{1,2,3,4}$ Communications and Electronic Department, Engineering College/ Arab Academy of Science and \\ Technology (AAST), Egypt
}

\begin{abstract}
Space communication systems as employed in satellite to satellite links are traditionally performed using microwaves. Since, the future of satellite systems will permit more efficient and more reliable operation these (Inter Satellite Links) ISLs will be optical links. This paper proposes a link model between satellites in free space over Low Earth Orbit (LEO) in order to recognize maximum bit rate. The performance of such a link is affected by various parameters, as in depending on the propagating wavelength. Moreover, the maximum bit rate is calculated and analyzed employing Avalanche Photodiode (APD) and Non Return to Zero (NRZ) modulation at different wavelengths improving the quality of the system.
\end{abstract}

Keywords: Free Space Optics (FSO); Optical Wireless Communication (OWC); Inters Satellite Link (ISL).

\section{Introduction}

The Optical Wireless Communication (OWC) system plays an important role in the architecture of hybrid wireless communications. Recently, Free Space Optics (FSO) is one of the OWC technologies and became more promising technology for Inter Satellite Links (ISLs) due to its higher speed, efficiency and reliability compared to microwave links as well it has a very large difference in wavelength [1]. Therefore, the OWC has attracted attention as next generation core technology for FSO communication networks using satellites which can be connected with bit rates up to numerous Gbps [2].

Space laser communication system operates in a short frequency range possesses many advantages such as: high bit transmit rate, high bandwidth, small optical antenna size and weight, narrow field of view, narrow laser emit beam, power efficiency, high precision and etc. [3].

However many challenges arise in FSO technology and limit its deployment in the communications field. These challenges are affecting on the communication link between the two transmitters whatever mobile or fixed link, and result in either disconnecting or attenuating the link. The challenges that face mobile FSO links are the variation in both receiver beam profile of the FSO link and received optical power due to the constantly changing transmitter receiver separation distance. However, in this paper the proposed link will neglect these changes assuming no vibrations [4].

Therefore, currently, static FSO links are more reliable than mobile FSO links because there are no continuous tracking and alignment requirements needed to maintain Line Of Sight (LOS) [4]. This LOS technology offers numerous advantages for both telecommunication users and providers. It provides a high bit rates up to several Gbps through different wavelengths. These wavelengths with their stipulations and considerations will be discussed later. This paper firstly makes an overview of proposed optical ISL system in section II. Then, the selection of different optical wavelengths which used for terrestrial as well as near-earth and specifically for deep space links is recorded in section III. Section IV is written to revise the ISL link performance expending system parameters focusing on transmitted bit rate between LEO satellites at bit error rate (BER) ranged from $10^{-6}$ to $10^{-9}$ [5][6][7], employing APD detector and NRZ modulation at different wavelengths maximizing the quality of the system as well. Then section V concludes the paper.

\section{Optical Isl System}

The optical system of ISL is consisting of two transceivers communicating by means of emitting and receiving optical signals. The proposed ISL uses lasers in free space channel as a signal carrier as well a wireless connectivity between transmitter and receiver as in Fig. 1. Using this FSO channel is considered a key technology for realizing an ultrahigh speed and long-haul communication system. This laser source output is transferred, and the laser beam formed by collimation lenses system, and emitted using an optical antenna. The optical path loss depends on the laser wavelength and distance between transmitter and receiver platforms [8].

At the receiver, the process is reversed and optical signal is converted into a bit stream. These bit streams modulated using pulse position modulation (PPM) or Non Return to Zero (NRZ) modulation. However this paper simulate the model using NRZ modulation, because is superlative scheme for obtaining maximum 
coverage distance of the link [9]. It becomes extremely difficult to modulate the laser directly; therefore Mach Zehnder (MZ) modulator used as an external and alternative optical modulator as seen in Fig. 1 [10].

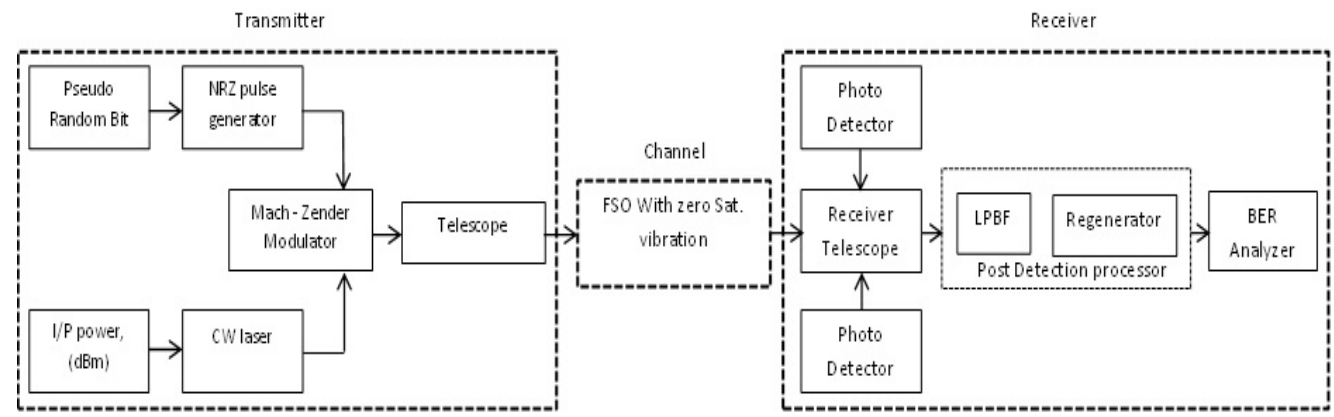

Fig. 1.ISL system

Since the performance of the system is significantly influenced by the propagation medium is more sufficient to characterize the link by its wavelength instead of its terahertz-frequency spectrum [11]. Thus, the selection of wavelength is preferable for FSO communication systems and it will be discussed in the next section.

\subsection{Wavelengths across the Spectrum}

\section{Wavelength Considerations}

Several transmission bands have been defined and standardized from the original O-band to the U/XLbands as seen in table 1 . The E- and U/XL-bands have typically been avoided because they correspond to high transmission loss regions. The E-band represents the water peak region, while the U/XL-band resides at the very end of the transmission window for silica glass [12].

TABLE I

Optical wavelength bands [12]

\begin{tabular}{|c|c|c|c|c|c|c|}
\hline Name & $\mathbf{O}$ & $\mathbf{E}$ & $\mathbf{S}$ & $\mathbf{C}$ & $\mathbf{L}$ & U/XL \\
\hline Wavelength & $1260-1360$ & $1360-1460$ & $1460-1530$ & $1530-1565$ & $1565-1625$ & $1625-1675$ \\
\hline Note & $\begin{array}{c}\text { Original } \\
\text { band }\end{array}$ & Water peak band & & $\begin{array}{l}\text { Bands us } \\
\text { perforn }\end{array}$ & $\begin{array}{l}\text { he higher } \\
\text { ystems }\end{array}$ & Not used \\
\hline
\end{tabular}

In addition to that the original fiber networks installed a couple of decades ago operated only at 1310 $\mathrm{nm}$ in the (O-band). Then the $1550 \mathrm{~nm}$ wavelength in the (S-band), which became along soon after, and both wavelengths formed the basis for the explosion in traffic in the transport portion of the network in the 1980s and 1990s [13]. The rest of bands used in Dense Wavelength Division Multiplexing (DWDM) applications operating in the (C and L - bands), which also became of age in the late 1990s and early part of the following decade.

Furthermore, optical carrier frequencies in the order of $200 \mathrm{THz}(1550 \mathrm{~nm})$ or $350 \mathrm{THz}(850 \mathrm{~nm})$ are free of any license requirements worldwide and cannot interfere with satellite or other Radio Frequency (RF) equipment. Nevertheless these bands weren't used only in optical fiber communications it became commercially available today in FSO systems that operate in the near infrared (IR) wavelength range between roughly 750 and $1600 \mathrm{~nm}$. Toward ease rapid installation, FSO systems operating in the near IR range can also be used for connections through glass [14].

Unfortunately, optical devices which use the $800 \mathrm{~nm}$ spectral range cannot operate above $2.5 \mathrm{Gbps}$ because of the power limitations imposed for eye safety. In order to overcome this bandwidth and power limitations, $1550 \mathrm{~nm}$ wavelength is selected for new ultra-high speed FSO systems [15].

\section{2. wavelength selection}

The selection of optical wavelength for FSO systems especially ISLs is primarily based on the optical transmission windows, eye safety reasons, of course expenses, atmospheric effects, the availability of receiver and transmitter components as well depending on the desired applications [16][17][18].

Since this paper mainly converse about ISL as an optical link, the using of these wavelengths (750 $850 \mathrm{~nm}$ ) might be available, because in space the eye safety precautions assumed to be zero. Therefore, these wavelengths are suitable for FSO operation, and several vendors provide higher power laser sources that operate in this region. The different wavelengths used in the proposed link are listed in table 2. 
These wavelengths are using commercially available detectors. This technology of detectors is typically fits into two categories. However, each type is working according to specific wavelength as in visible (specifically, 400-1000 nm) and IR (950-1650 nm) wavelengths. Visible wavelengths are typically serviced by sensitive silicon ( $\mathrm{Si}$ ) APD technology, as well in $850 \mathrm{~nm}$ wavelength which has reliable, inexpensive, highperformance transmitter and is readily available and commonly used in network and transmission equipment [19]. IR wavelengths are serviced by either InGaAs APDs or, recently, by superconducting single photon detectors [20]. These entire previous wavelengths also can be categorized into different bands otherwise called optical spectrum as seen in table 2 .

\section{TABLE II}

Optical bands[21]

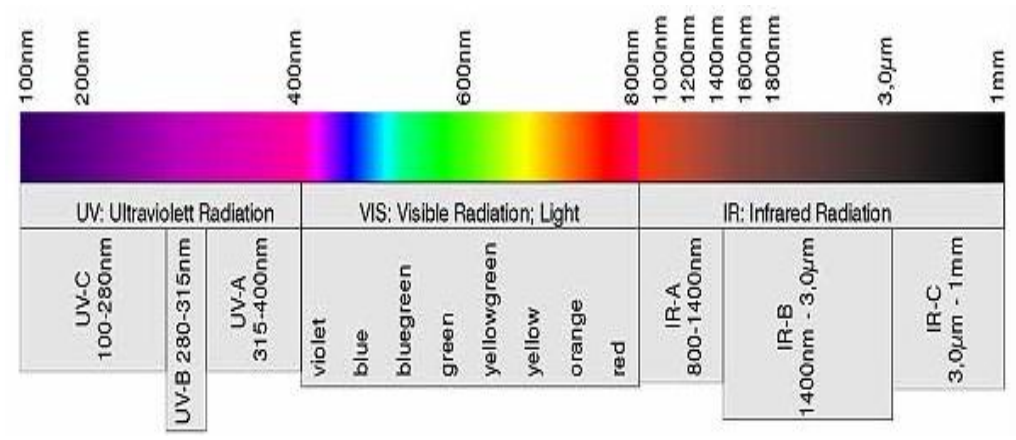

A comparison on the performance of ISL as a function of system parameters operating in different wavelengths obtaining allowable transmitted bit rate in each band at maximum BER ranged from $10^{-6}$ to $10^{-9}$ is investigated in results section.

\section{Results And Discussion}

The analysis of the link performance corresponding to the wavelength selection is obtained by simulated link using optisystem Ver.7 as shown in fig. 2. The ISL is supported by various parameters as listed in table 3 such as $12 \mathrm{dBm}$ input power that provides transmission between satellites over a distance of several tens of thousands of kilometers [22]. However, this research will focus on bit rate transferred between LEO links.

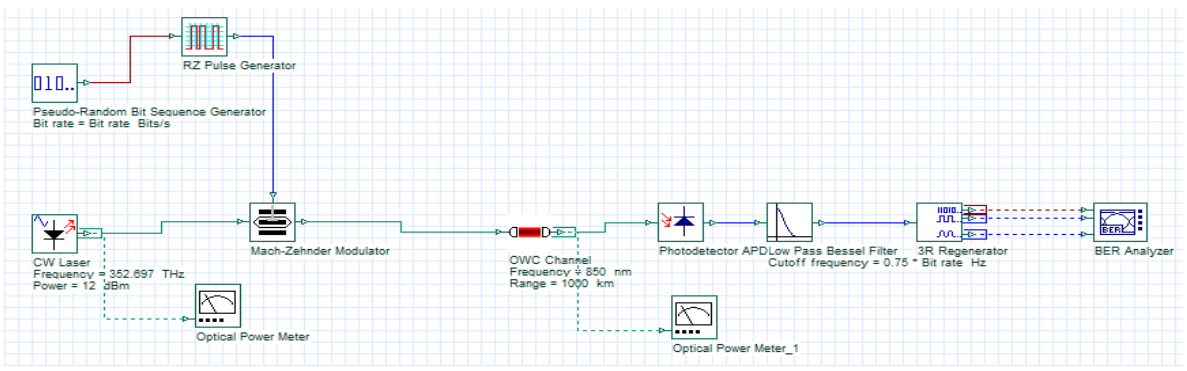

Fig. 2.Proposed ISL

TABLE III

Simulation parameters of ISL system

\begin{tabular}{|l|c|}
\hline \multicolumn{1}{|c|}{ Parameters specifications } & value \\
\hline OWC parameters \\
\hline Transmit power & LOS \\
\hline Modulation type & $12 \mathrm{dBm}$ \\
\hline Extinction ratio of MZ modulator & NRZ \\
\hline Transmitter aperture diameter & $26 \mathrm{~dB}$ \\
\hline Receiver aperture diameter & $15 \mathrm{~cm}$ \\
\hline Detector type & APD \\
\hline Gain & 3 \\
\hline Responsivity & $1 \mathrm{~A} / \mathrm{W}$ \\
\hline Dark current & $10 \mathrm{nA}$ \\
\hline \multicolumn{2}{|c|}{ Variable parameters } \\
\hline Propagation distance & $1000-3000 \mathrm{~km}$ \\
\hline Wavelength & Table 1,2 \\
\hline Bit rate & $2.5-40 \mathrm{Gbps} / 1000 \mathrm{~km}$ \\
\hline Minimum received power & Table 5 \\
\hline
\end{tabular}


For the reason that the currently typical channel capacity for optical fiber transmission is from $2.5 \mathrm{Gbps}$ to $40 \mathrm{Gbps}$ which is high speed transmission. Became there is a need for a flexible full FSO transmission system which can provide capacity equivalent to optical fiber [15]. Thus, fixed distance have been chosen for LEO orbit to be $1000 \mathrm{~km}$ with bit rate in channel capacity for transmission is from 2.5 to $40 \mathrm{Gbps}$, and for $3000 \mathrm{~km} \mathrm{LEO}$ distance the transmitted bit rate ranged from $15 \mathrm{Mbps}$ to $5.6 \mathrm{Gbps}$. The varying in wavelength will be according to each band as mentioned in tables 1 and 2 .

In first circumstance after simulating the link using the most common wavelength in FSO systems which is $1550 \mathrm{~nm}$ (C-band), investigated that the maximum bit rate could be sent over $1000 \mathrm{~km}$ is about $3 \mathrm{Gbps}$ with Q-factor about 4.5 corresponding to BER $10^{-6}$ as shown in Fig. 3, and all these values is not consider optimum values for describing system performance.

Otherwise, in case of increasing the wavelength to higher bands as in L-band spotted that the system performance decreased as in Fig. 4 instead of Q-factor because of reducing the size of eye-opening which increase the potential occurrence for bit errors and jitter as well.

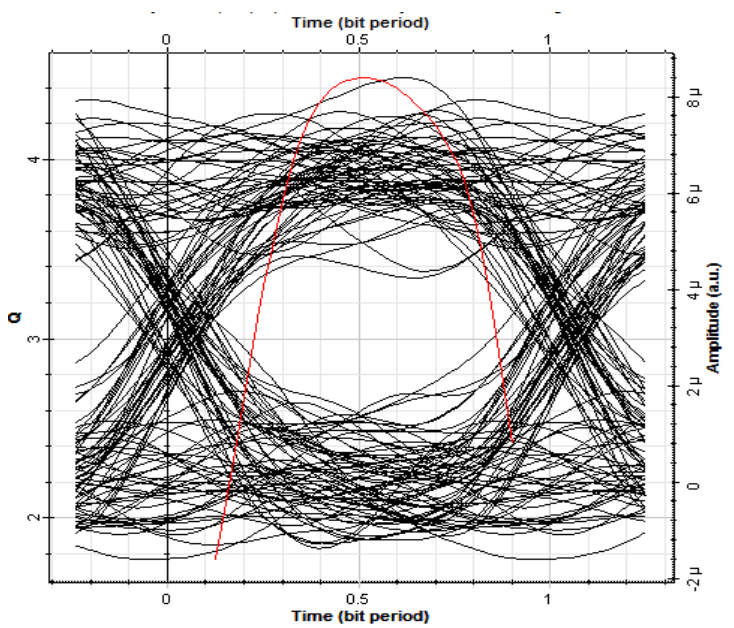

Fig. 3.Eye diagram for Q- Factor at (C- band) at $3 \mathrm{Gbps}$ over $1000 \mathrm{~km}$

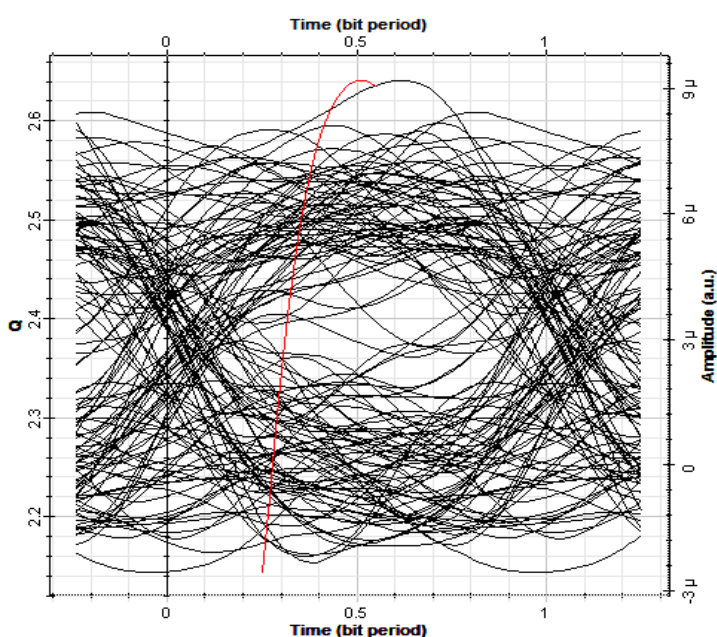

Fig. 4.Eye diagram for $\mathrm{Q}$-factor at (L- band) at $3 \mathrm{Gbps}$ over $1000 \mathrm{~km}$

Then, through decreasing the wavelength to E-band noted that the system performance interms of the Q-factor is increased compared to higher bands. However, in transmitting higher bit rate the quality of the system decreased for the same band as in Fig. 5. Also observed from that figure the Q- factor is inversely proportional with bit rate in the same wavelength, and that bit rate is in direct proportional relevant to the BER.

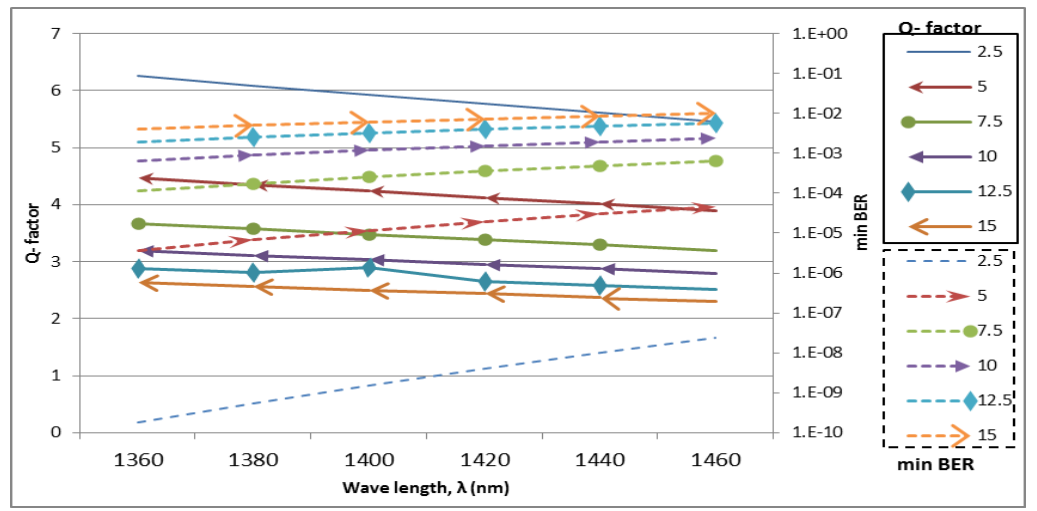

Fig. 5.Q-factor and min- BER in relation with (E-band) wavelengths at different bit rate

Concerning to change the wavelength to lower bands as in O-bands observed that at transmitting 2.5 Gbps selecting $1300 \mathrm{~nm}$ wavelength the Q-factor of the system improved with $10^{-12}$ BER as seen in fig. 6 . Hence, in case of decreasing the wavelength the system performance increased instead of Q- factor with reducing BER as well. 


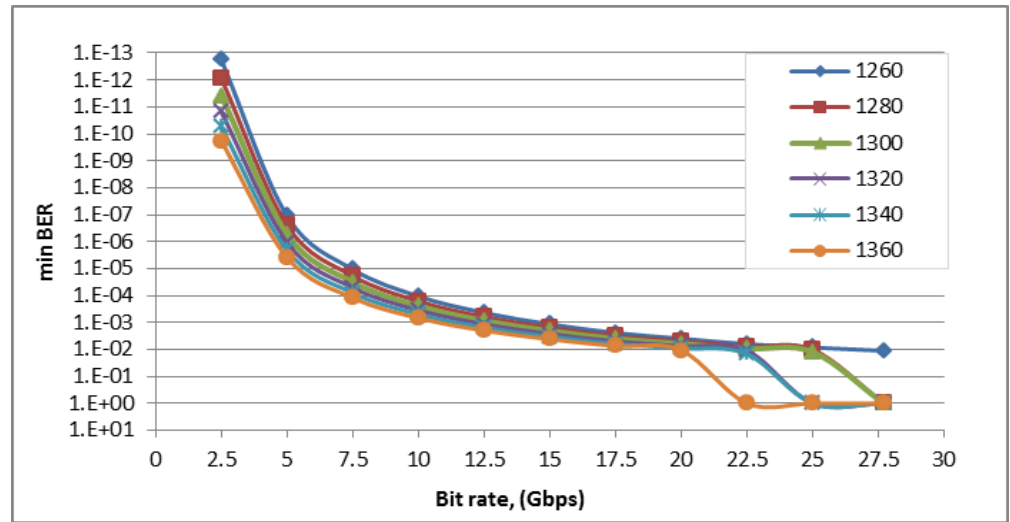

Fig.6. min - BER in relation with bit rate at different (O-band) wave lengths

In addition to another circumstance by selecting visible light (VIS) otherwise near to visible light (NVIS) bands they are considered the preeminent selection for the wavelength in space, because the Q- factor of the system as shown in fig. 7 is about 60 which is an appropriate value to optimize the system performance given zero min BER as shown in Fig. 8 and 9.

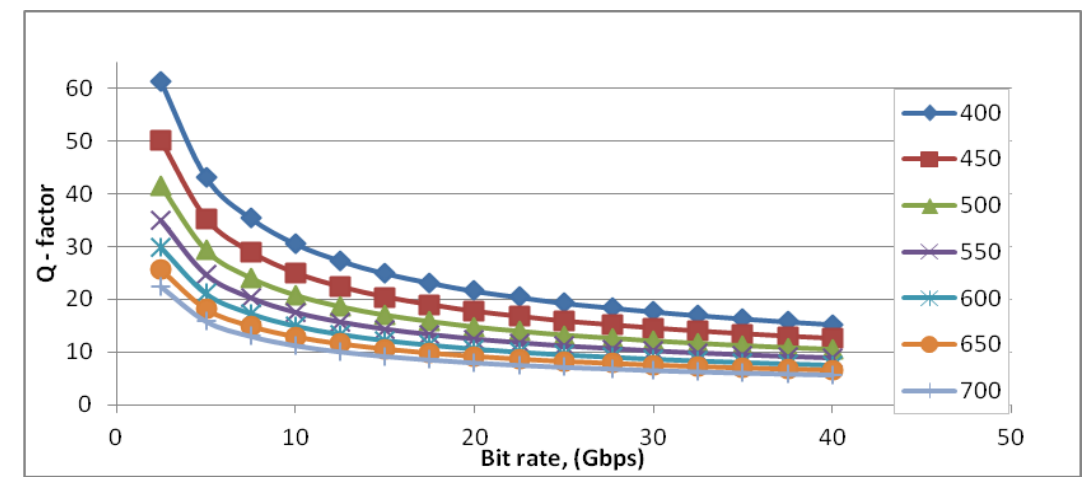

Fig. 7.Q- Factor in relation with bit rate at different wave length (VIS-band)

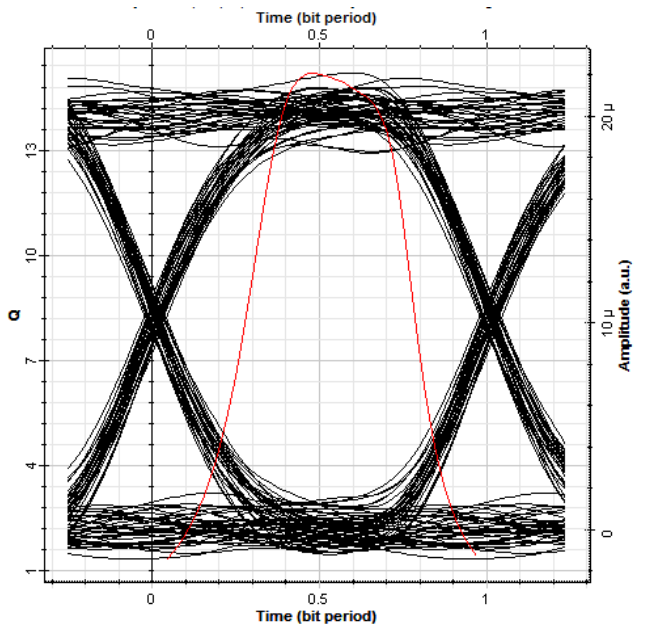

Fig. 8.Eye diagram for $\mathrm{Q}-$ factor using (NVIS-band) at $2.5 \mathrm{Gbps}$

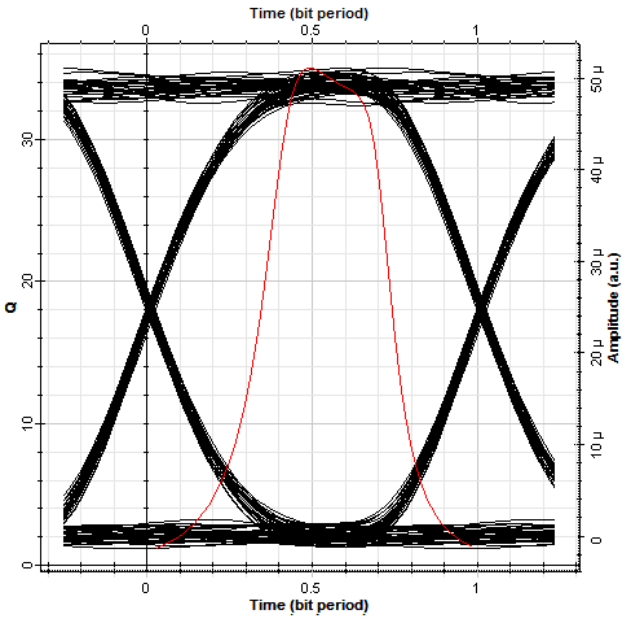

Fig. 9.Eye diagram for min-BER using (VIS-band) at $2.5 \mathrm{Gbps}$

Furthermore, after investigating the simulated link performance, the allowable transmitted bit rate over $1000 \mathrm{~km}$ as well $3000 \mathrm{~km}$ LEO distances in different bands have been calculated corresponding to maximum BER $10^{-6}$ to $10^{-9}$ [7] and listed in table 4 . Then, from cited table observed that the $3 \mathrm{Gbps}$ bit rate could be transmitted over different bands achieving $10^{-6}$ BER depending on desired application, if it is obligatory to send $3 \mathrm{Gbps}$ over $3000 \mathrm{~km}$ distance in application with eye safety limitations the(C-band) might be used, otherwise as in this paper by neglecting the eye safety precautions the (VIS-band) could be used. Similarly, 2 Gbps could be transmitted achieving $10^{-9}$ BER at many bands. 
The Influence of Varying the Optical Wavelength on ISL Performance Recognizing High Bit Rates

TABLE IV Comparison between allowable transmitted bitrate over different LEO distances

\begin{tabular}{|c|c|c|c|c|c|}
\hline \multirow{2}{*}{ Band } & \multirow{2}{*}{$\begin{array}{l}\text { Selected wave } \\
\text { length }\end{array}$} & \multicolumn{2}{|c|}{ Bitrate at BER $10^{-6}$} & \multicolumn{2}{|c|}{ Bitrate at BER $10^{-9}$} \\
\hline & & $1000 \mathrm{~km}$ & $3000 \mathrm{~km}$ & $1000 \mathrm{~km}$ & $3000 \mathrm{~km}$ \\
\hline Visible light & \multirow{2}{*}{500} & \multirow{2}{*}{ *40 Gbps } & \multirow{2}{*}{$\begin{array}{c}3 \\
\text { Gbps }\end{array}$} & \multirow{2}{*}{$* 40$} & \multirow{2}{*}{$\begin{array}{c}2 \\
\text { Gbps }\end{array}$} \\
\hline $400-700$ & & & & & \\
\hline Near Visible & \multirow{2}{*}{860} & \multirow{2}{*}{$\begin{array}{c}33 \\
\text { Gbps }\end{array}$} & \multirow{2}{*}{$\begin{array}{l}420 \\
\text { Mbps }\end{array}$} & \multirow{2}{*}{$\begin{array}{c}18 \\
\text { Gbps }\end{array}$} & \multirow{2}{*}{$\begin{array}{l}225 \\
\text { Mbps }\end{array}$} \\
\hline $800-920$ & & & & & \\
\hline $\mathrm{O}-$ band & \multirow{2}{*}{1300} & \multirow{2}{*}{$\begin{array}{c}6.5 \\
\text { Gbps }\end{array}$} & \multirow{2}{*}{$\begin{array}{c}81 \\
\text { Mbps }\end{array}$} & \multirow{2}{*}{$\begin{array}{c}3.7 \\
\text { Gbps }\end{array}$} & \multirow{2}{*}{$\begin{array}{c}45 \\
\text { Mbps }\end{array}$} \\
\hline $1260-1360$ & & & & & \\
\hline E-band & \multirow{2}{*}{1440} & \multirow{2}{*}{$\begin{array}{c}4 \\
\text { Gbps }\end{array}$} & \multirow{2}{*}{$\begin{array}{c}53 \\
\text { Mbps }\end{array}$} & \multirow{2}{*}{$\begin{array}{l}2.25 \\
\text { Gbps }\end{array}$} & \multirow{2}{*}{$\begin{array}{c}30 \\
\text { Mbps }\end{array}$} \\
\hline $1360-1460$ & & & & & \\
\hline $\mathrm{S}-$ band & \multirow{2}{*}{1500} & \multirow{2}{*}{$\begin{array}{c}3.5 \\
\text { Gbps }\end{array}$} & \multirow{2}{*}{$\begin{array}{c}46 \\
\text { Mbps }\end{array}$} & \multirow{2}{*}{$\begin{array}{c}2 \\
\text { Gbps }\end{array}$} & \multirow{2}{*}{$\begin{array}{c}26 \\
\text { Mbps }\end{array}$} \\
\hline $1460-1530$ & & & & & \\
\hline $\mathrm{C}-$ band & \multirow{2}{*}{1550} & \multirow{2}{*}{$\begin{array}{c}3 \\
\text { Gbps }\end{array}$} & \multirow{2}{*}{$\begin{array}{c}40 \\
\text { Mbps }\end{array}$} & \multirow{2}{*}{$\begin{array}{l}1.75 \\
\text { Gbps }\end{array}$} & \multirow{2}{*}{$\begin{array}{l}22.5 \\
\text { Mbps }\end{array}$} \\
\hline $1530-1565$ & & & & & \\
\hline $\mathrm{L}$ - band & \multirow{2}{*}{1615} & \multirow{2}{*}{$\begin{array}{l}2.75 \\
\text { Gbps }\end{array}$} & \multirow{2}{*}{$\begin{array}{c}34 \\
\text { Mbps }\end{array}$} & \multirow{2}{*}{$\begin{array}{c}1.5 \\
\text { Gbps }\end{array}$} & 19.5 \\
\hline $1565-1625$ & & & & & Mbps \\
\hline
\end{tabular}

(*) This value is the practical value which could be sending over this distance, and the theoretical value is $250 \mathrm{Gbps}$ but cannot be sent because of the maximum value of the MZ modulator to convert is $40 \mathrm{Gbps}$.

Consequently, selecting $850 \mathrm{~nm}$ wavelength as one of the (NVIS-band) confirms why several vendors provide to operate in this region and became commonly used in the most of FSO applications in space. Therefore, as stated before there is no concern in selection VIS or NVIS bands in space since there is no need for eye safety limitations.

After comparing the simulated link at different optical bands, this paper established that the received power is constant at each band at any bit rate as listed in table 5, and it is in direct proportional with the wavelength as shown in fig. 10.

TABLE V Received power at different bands at any transmitted bit rate

\begin{tabular}{|c|c|c|c|}
\hline \multirow{2}{*}{ Band } & \multirow{2}{*}{ Selected wavelength } & \multicolumn{2}{|c|}{ Received power } \\
\hline & & 1000 km & $3000 \mathrm{~km}$ \\
\hline Visible light & \multirow{2}{*}{500} & \multirow{2}{*}{-20.252} & \multirow{2}{*}{-29.794} \\
\hline $400-700$ & & & \\
\hline Near Visible & \multirow{2}{*}{860} & \multirow{2}{*}{-24.962} & \multirow{2}{*}{-34.505} \\
\hline $800-920$ & & & \\
\hline $\mathrm{O}-$ band & \multirow{2}{*}{1300} & \multirow{2}{*}{-28.551} & \multirow{2}{*}{-38.094} \\
\hline $1260-1360$ & & & \\
\hline E-band & \multirow{2}{*}{1440} & \multirow{2}{*}{-29.44} & \multirow{2}{*}{-38.983} \\
\hline $1360-1460$ & & & \\
\hline $\mathrm{S}-$ band & \multirow{2}{*}{1500} & \multirow{2}{*}{-29.794} & \multirow{2}{*}{-39.337} \\
\hline $1460-1530$ & & & \\
\hline $\mathrm{C}-$ band & \multirow{2}{*}{1550} & \multirow{2}{*}{-30.097} & \multirow{2}{*}{-39.621} \\
\hline $1530-1565$ & & & \\
\hline $\mathrm{L}-$ band & \multirow{2}{*}{1615} & \multirow{2}{*}{-30.436} & \multirow{2}{*}{-39.978} \\
\hline $1565-1625$ & & & \\
\hline
\end{tabular}

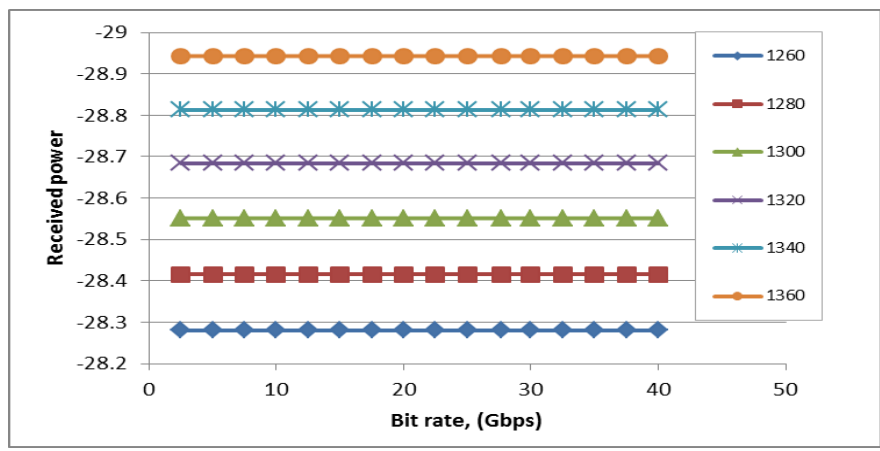

Fig. 10.Received power in relation with bit rate at different wave length (O-band) 


\section{Conclusion}

This paper has been focused on the impact of wavelength variation on proposed ISL between two satellites network at LEO distance using APD detector and NRZ modulation. This research concludes that the exceptional selection for optical bands are VIS or NVIS bands rather than the most commonly used (C-band), to be used in FSO systems for realizing adequate system performance instead of Q- factor with zero BER. Also, the paper calculated and analyzed the allowable high bit rate resulted at maximum BER ranged from $10^{-6}$ to $10^{-9}$, and determined that the same value of resulted bit rate might be send over different bands at same BER in different distances depending on desired applications. Moreover, this research detected that the received power is constant at each band at any transmitted bit rate.

\section{References}

[1] A. H. Hashim, F. D. Mahad, S. M. Idrus, A. Sahmah, M. Supa, and P. T. Centre, Modeling and Performance Study of Inter Satellite Optical Wireless Communication Syste, Vol.64,2010.

[2] F. Heine, H. Kämpfner, and R. Lange, Optical Inter-Satellite Communication Operational,2010, pp. 1583-1587.

[3] K. Shantha lakshmi, M. P. Senthil kumar, and K. V. N. Kavitha, "Inter-satellite laser communication system," 2008 Int. Conf. Comput. Commun. Eng., , May 2008., pp. 522-527.

[4] M. K. Al-Akkoumi, A. Harris, R. C. Huck, and J. J. Sluss, Jr., Challenges facing mobile free-space optical communications, vol. 7324, May 2009., pp. 73240L-73240L-11.

[5] B. Barua, Comparison the Performance of Free-Space Optical Communication with OOK and BPSK Modulation under Atmospheric Turbulence, vol. 3, no. 5,2011, pp. 4391-4399.

[6] B. Smutny, R. Lange, H. Kämpfner, D. Dallmann, G. Mühlnikel, M. Reinhardt, K. Saucke, U. Sterr, B. Wandernoth, and R. Czichy, In-orbit verification of optical inter-satellite communication links based on homodyne BPSK</title $>$," vol. 6877, Feb 2008, pp. 687702-687702-6.

[7] J. Takashi, Optical Inter-orbit Communication Experiment between OICETS and ARTEMIS, , 2006, pp. 23-33.

[8] K. Shantha lakshmi, M. P. Senthil kumar, and K. V. N. Kavitha, "Inter-satellite laser communication system, 2008 Int. Conf. Comput. Commun, , May 2008, pp. 522-527.

[9] B. Patnaik and P. K. Sahu, Inter-satellite optical wireless communication system design and simulation, IET Commun., vol. 6, no. 16, , Nov. 2012, pp. 2561-2567.

[10] M. Schemes, Tutorial Note \# 5 Modulation Schemes, SHF Communication Technologies AG. Germany.

[11] M. Achour, D. Ph, S. Hwy, and S. Beach, Free-Space Optics Wavelength Selection : $10 \mu$ Versus Shorter Wavelengths, pp. 1-15.

[12] O. Bands, B. Laurent, and G. Draka, From O to L: The Future of optical wavelength-bands, June , 2008, pp. 83-85.

[13] M. Boxer, Full Spectrum Fiber Comes of Age,OFS, 2012

[14] M. Gebhart, P. Schrotter, U. Birnbacher, E. Leitgeb, and A. S. C. Satcom, Satellite Communications , Free Space Optics and Wireless LAN combined: Worldwide broadband wireless access independent of terrestrial infrastructure, IEEE MELECON 2004, , Dubrovnik, Croati, May 12-15,2004.

[15] T. Studies, Studies on Performance of Ultra High Speed Free-Space Optical Communication Systems, School of Global Information and Telecommunication Studies, Waseda University, February, 2007.

[16] T. Plank, E. Leitgeb, and M. Loeschnigg, Recent developments on free space optical links and wavelength analysis, 2011 Int. Conf. Sp. Opt. Syst. Appl, May 2011, pp. 14-20.

[17] T. Plank, M. Czaputa, E. Leitgeb, S. S. Muhammad, N. Djaja, B. Hillbrand, P. Mandl, and M. Schönhuber, wavelength selection on fso-links, 2011, pp. 2508-2512.

[18] Sanka Gateva, Photodetectors ( InTech, March 23, 2012 ).

[19] S. Bloom, E. Korevaar, and J. Schuster, Understanding the performance of free-space optics [ Invited ], vol. 2, no. 6, 2003, pp. 178-200.

[20] J. Bourgoin, B. L. Higgins, B. Helou, R. Girard, R. Laflamme, and T. Jennewein, A comprehensive design and performance analysis of LEO satellite quantum communication, 2012, pp. 1-31.

[21] The Wavelength Range Of Optical Radiation.htm , 2013

[22] Singh, Kuldeepak, performance improvement of intersatellite optical wireless communication with multiple transmitter and receivers, Vol. 1 Issue 4, June 2012. 